\title{
Identification of candidate genes of QTLs for seed weight in Brassica napus through comparative mapping among Arabidopsis and Brassica species
}

\author{
Guangqin Cai ${ }^{1,2}$, Qingyong Yang ${ }^{1}$, Qian Yang ${ }^{1}$, Zhenxing Zhao ${ }^{1,2}$, Hao Chen ${ }^{1}$, Jian Wu', Chuchuan Fan ${ }^{1}$ \\ and Yongming Zhou ${ }^{1,2^{*}}$
}

\begin{abstract}
Background: Map-based cloning of quantitative trait loci (QTLS) in polyploidy crop species remains a challenge due to the complexity of their genome structures. QTLs for seed weight in B. napus have been identified, but information on candidate genes for identified QTLs of this important trait is still rare.

Results: In this study, a whole genome genetic linkage map for B. napus was constructed using simple sequence repeat (SSR) markers that covered a genetic distance of 2,126.4 cM with an average distance of $5.36 \mathrm{cM}$ between markers. A procedure was developed to establish colinearity of SSR loci on B. napus with its two progenitor diploid species B. rapa and B. oleracea through extensive bioinformatics analysis. With the aid of B. rapa and B. oleracea genome sequences, the 421 homologous colinear loci deduced from the SSR loci of B. napus were shown to correspond to 398 homologous loci in Arabidopsis thaliana. Through comparative mapping of Arabidopsis and the three Brassica species, 227 homologous genes for seed size/weight were mapped on the B. napus genetic map, establishing the genetic bases for the important agronomic trait in this amphidiploid species. Furthermore, 12 candidate genes underlying 8 QTLs for seed weight were identified, and a gene-specific marker for BnAP2 was developed through molecular cloning using the seed weight/size gene distribution map in B. napus.

Conclusions: Our study showed that it is feasible to identify candidate genes of QTLs using a SSR-based B. napus genetic map through comparative mapping among Arabidopsis and B. napus and its two progenitor species B. rapa and $B$. oleracea. Identification of candidate genes for seed weight in amphidiploid $B$. napus will accelerate the process of isolating the mapped QTLs for this important trait, and this approach may be useful for QTL identification of other traits of agronomic significance.
\end{abstract}

Keywords: Brassicaceae, Rapeseed, Arabidopsis, Comparative mapping, QTL, Map-based cloning, Seed weight

\section{Background}

Rapeseed (Brassica napus L., AACC, $2 \mathrm{n}=38$ ) is one of the world's most important oil crops and provides not only edible oil for human diets, but also protein-rich feed for animals and raw materials for industrial processes such as biodiesel production. B. napus is an amphidiploid species derived from the hybridization of its two diploid progenitor species, $B$. rapa (AA, $2 \mathrm{n}=20$ )

\footnotetext{
* Correspondence: ymzhou@mail.hzau.edu.cn

'National Key Laboratory of Crop Genetic Improvement, Huazhong Agricultural University, Wuhan, 430070, China

${ }^{2}$ Key Laboratory of Rapeseed Genetics and Breeding of Agriculture Ministry of China, Huazhong Agricultural University, Wuhan 430070, China
}

and B. oleracea $(\mathrm{CC}, 2 \mathrm{n}=18)$ [1]. Studies have shown that Arabidopsis thaliana, B. napus, B. rapa and B. oleracea have a common ancestor [2-6].

Seed weight is one of the three yield components (siliques per plant, seeds per silique and seed weight) of plant productivity of rapeseed and is also related to oil and protein content [7-9]. Extensive efforts have been made in mapping of the QTLs for seed size/weight in crop species [10-14], and genes governing seed size/ weight have been cloned in model plants Arabidopsis and rice through mutant analysis and map-based cloning [15-18]. However, molecular cloning of seed size/weight genes in other crops, such as rapeseed, wheat and

\section{Biomed Central}


soybean lags behind due to the more complicated genome structures of these crops and limited availability of genome sequence information.

Quantitative genetic analysis in B. napus showed that seed weight has a relatively high heritability and may primarily be controlled by genes with additive effects [11,14,19-21]. Quijada et al. [22] detected three QTLs (located on N7, N17 and N19, respectively) for seed weight in different populations and environments, but no common QTL was identified. Udall et al. [23] found 6, 4 and 5 QTLs of seed weight in Hua DH, SYN DH and testcross populations, respectively, with only one QTL (located on N14) detected in all populations and environments. Shi et al. [21] mapped 159 QTLs of seed weight in $\mathrm{TN} \mathrm{DH}$ and $\mathrm{RC}-\mathrm{F}_{2}$ populations across 10 environments with only one major QTL (qSW.A7-2) identified in all environments. In our previous study [11], 9 QTLs for seed weight in a doubled haploid (DH) population of $B$. napus were identified, among which two major QTLs, TSWA7a and TSWA7b, were stably detected across years. Interestingly, seed weight QTLs on A7 were repeatedly detected in other studies with diverse genetic materials $[11,14,21,24]$. However, little is known about the candidate genes for those mapped QTLs, and so far only two genes, BnMINI3a and $B n T T G 2 a$, were assigned as the candidate genes for $T S W A 5 b$ and TSWA5c [11]. It is thus crucial to develop procedures that can accelerate the process of mappedbased cloning by identification of candidate genes of those QTLs.

Comparative mapping among related species is a powerful tool for genetic studies by offering the possibility of transferring genomic information from wellstudied species to more genetically complicated ones. This advantage is particularly obvious in Brassicaceae, because Arabidpsis thaliana, as a model plant for dicots, has completed genome sequence [25], and a wealth of functional genomics information. Much effort has been focused on the comparative analysis between Brassica species and $A$. thaliana. Several comparative maps in Brassicaceae have been constructed based on RFLP markers, cDNA clones from Brassica species [2,26-32]. Other types of markers, such as IP (intron polymorphism) markers from Arabidopsis [33], and gene specific markers based on Arabidopsis sequences were also used [34]. So far, no comparative mapping with Arabidopsis has been conducted with genetic maps based on SSR markers or other unknown sequence markers.

Wang et al. [32] constructed an integrated linkage map of $B$. napus using mainly SSR markers with the aid of other type of markers including RFLP, and then attempted to identify homologous loci in Arabidopsis to these SSR markers. However, $<2 \%$ of the primer pairs had homology in Arabidopsis, of which only 50\% agreed with those identified using the corresponding SSR clone sequences. In such a case, it was difficult for comparative studies within Brassicaceae only based on the SSR marker primer sequences [32]. On the other hand, in a comparative study based on 6, 5 and 6 BACs' sequences from $B$. napus, $B$. rapa, and $B$. oleracea, respectively, it was found that most homologous loci in $\mathrm{A}$ and $\mathrm{C}$ genomes of B. napus, B. rapa and B. oleracea have colinear relationship with the same loci in Arabidopsis [35]. This finding implies that using the SSR markers homologous loci information in the B. rapa and B. oleracea genomes may bridge the comparative analysis of $B$. napus and Arabidopsis.

In order to transfer the gene information effectively from Arabidopsis to B. napus, we developed a procedure for comparative mapping among three Brassica species (B. napus, B. rapa and B. oleracea) and Arabidopsis based on a SSR linkage map in B. napus. By making use of the map, we identified the putative genes involved in seed weight/size regulation in B. rapa and B. oleracea, and mapped these genes onto the SSR-based B. napus genetic map. Such a seed weight/size gene distribution map will allow us to pinpoint candidate genes underlying seed weight QTLs, thus facilitating the genetic and molecular studies of seed weight control.

\section{Methods}

\section{Plant materials and phenotypic evaluation}

A DH population of 190 lines was produced from microspore culture with a $F_{1}$ cross between SW Hickory (a spring-type $B$. napus variety) and JA177 (a winter-type B. napus pure line), and named the SJ-DH population. The population was used for genetic and QTL mapping. Seed weight of each plant from the population was measured based on 500 fully developed seeds with three replications. The average seed weight was converted to 1000-seed weight (TSW) for each individual plant. The means of TSW of 10-15 plants from each plot were used for trait evaluation of parents, F1 and SJ DH lines. The detailed information for the production of the population, field trials and sampling procedures for seed weight measurement has been described previously [11].

\section{Molecular marker, linkage map and QTL mapping}

Primer sequences for SSR markers used for genetic mapping were described by Fan et al. [11] and the sequence information of newly added SSR markers is provided in Additional file 1: Table S1. Linkage analysis with all markers was performed using MAPMAKER 3.0 [36]. A minimum log likelihood of the odds (LOD) score of 11.0 and a maximum distance of $25 \mathrm{cM}$ were used to group loci into linkage groups (LGs). Genetic distances between SSR loci were calculated using the Kosambi mapping function. The nomenclature of LGs follows the 
rules proposed by the Multinational Brassica Genome Project [37]. QTLs were detected using the composite interval mapping (CIM) procedure with the software QTL Cartographer V2.5 [38]. The parameters and methods for QTL mapping were described as Fan et al. [11].

\section{Identification of homologous colinear loci in B. rapa and $B$. oleracea genomes through e-PCR amplification with SSR primers from $B$. napus}

To align the SSR loci on each of the $B$. napus LGs to the homologous loci in the $B$. rapa or B. oleracea genome, electronic PCR (e-PCR) [39] was performed with the primers of the SSR markers mapped on the B. napus LGs and the genomic sequences of $B$. rapa (version 1.1) [6,40] and B. oleracea (version 2011-06-30) [41] as templates. The parameters for e-PCR were set to allow three mismatches and one gap for a given primer pair. Amplicons produced from the e-PCR then were analyzed to determine their colinear relationship between the B. napus LGs and the chromosomes of $B$. rapa/B. oleracea.

An amplicon is regarded as a putative homologous colinear locus on the A- (B. rapa) or C- (B. oleracea) genome to the locus defined by the SSR marker used for the amplification on a particular B. napus LG. A homologous colinear locus was determined when only three or more amplicons on a same chromosome of B. rapa/ B. oleracea could be generated with the SSR primers from a single LG of $B$. napus. Such a criterion would allow to reduce the non-specific alignment among a LG in the genome of $B$. rapa or $B$. oleracea, and to determine the orientation of a linear fragment, which is required to establish the corresponding linear relationship between a $B$. napus LG and a B. rapa/B. oleracea chromosome.

To facilitate the process of identifying homologous colinear loci, a Perl script called e-PCRmap (Additional file 2) was written to analyze the results of e-PCR using the following formula:

$$
M_{x y}=\sum_{k=1}^{n} L_{y k}
$$

where $L$ is the variable describing the status of e-PCR amplification, while $M$ is the number of markers that fall onto the chromosomes of $B$. rapa and B. oleracea (with successful amplification), $\mathrm{x}$ is the LG of B. napus, and $\mathrm{y}$ is the chromosome of $B$. rapa and B. oleracea, $\mathrm{k}$ is the marker index of each LG, and $\mathrm{n}$ is the markers number of each LG. When a marker (k) on a LG (x) has one or more amplifications on a particular chromosome (y), $L_{\mathrm{yk}}$ is assigned 1, otherwise $L_{\mathrm{yk}}$ assigned 0 . The formula calculates how many possible amplicons are produced with the primers for the SSR markers in a particular LG.

The script generates a list of possible homologous colinear loci on the chromosomes of $B$. rapa or $B$. oleracea for each linkage group of B. napus, the order of the homologous colinear loci were the same to SSR loci distribution on the LG. When a marker had multiple amplification loci on a same chromosome, the accurate position for a particular locus was determined manually by referring to the physical positions of its upstream and downstream amplicons.

\section{Mapping Arabidopsis homologous loci onto B. napus genome}

The Perl script described above was used to extract the sequences of effective amplicons in the $B$ rapa and $B$. oleracea genomes. The amplicons' sequences were used as queries in searching for Arabidopsis homologues using the BLASTn program [42] against TAIR10 [43] with an E-value of 1.0 as an initial identification of homologous loci in Arabidopsis. The less stringent E-value could allow more homologous loci included for the identification of conserved blocks.

The positions and gene loci of best-hits in Arabidopsis genome sequences database were collected and comparatively mapped onto the $B$. napus LGs. Identification of the conserved Arabidopsis genomic blocks [5] on the B. napus LGs was performed essentially as described by Parkin et al. [30]. A conserved block contained a minimum of three mapped SSR loci with at least two homologous loci from one of the 24 defined Arabidopsis bocks [5] every $10 \mathrm{cM}$ in the $B$. napus genetic map.

\section{Identification of putative seed size/weight genes in $B$. napus}

To identify and locate the putative seed size/weight genes in $B$. napus, the homologous sequences of seed size/ weight genes in the databases of $B$. rapa and $B$. oleracea $[40,41]$ were first searched with the BLASTn [42] program (E value $<1 \mathrm{E}-20$ when using Arabidopsis seed size genes as query, and $\mathrm{E}$ value $<1 \mathrm{E}-10$ with the genes from other crops as query). The resulting sequences from the search were firstly mapped onto the chromosomes of $B$. rapa or $B$. oleracea and then placed on the B. napus LGs based on the homologous colinear relationships between $B$. napus and $B$. rapa/B. oleracea.

\section{Gene cloning}

Standard molecular cloning procedures [44] were followed to isolate the homologous genes of Arabidopsis in the parental lines of the SJ DH population. The genomic fragments corresponding to the AtAP2 gene were amplified using the primer pairs of AP2F (5'ATGTGGGATCTAAACGACTCACCA-3') and AP2R (5'TCAAGAAGGTCTCATGAGAGAAGG-3'). The PCR products from the parental lines were sequenced by the BigDye Terminator Cycle Sequencing v3.1 (Applied Biosystems, Foster City, CA, USA), and the coding sequences 


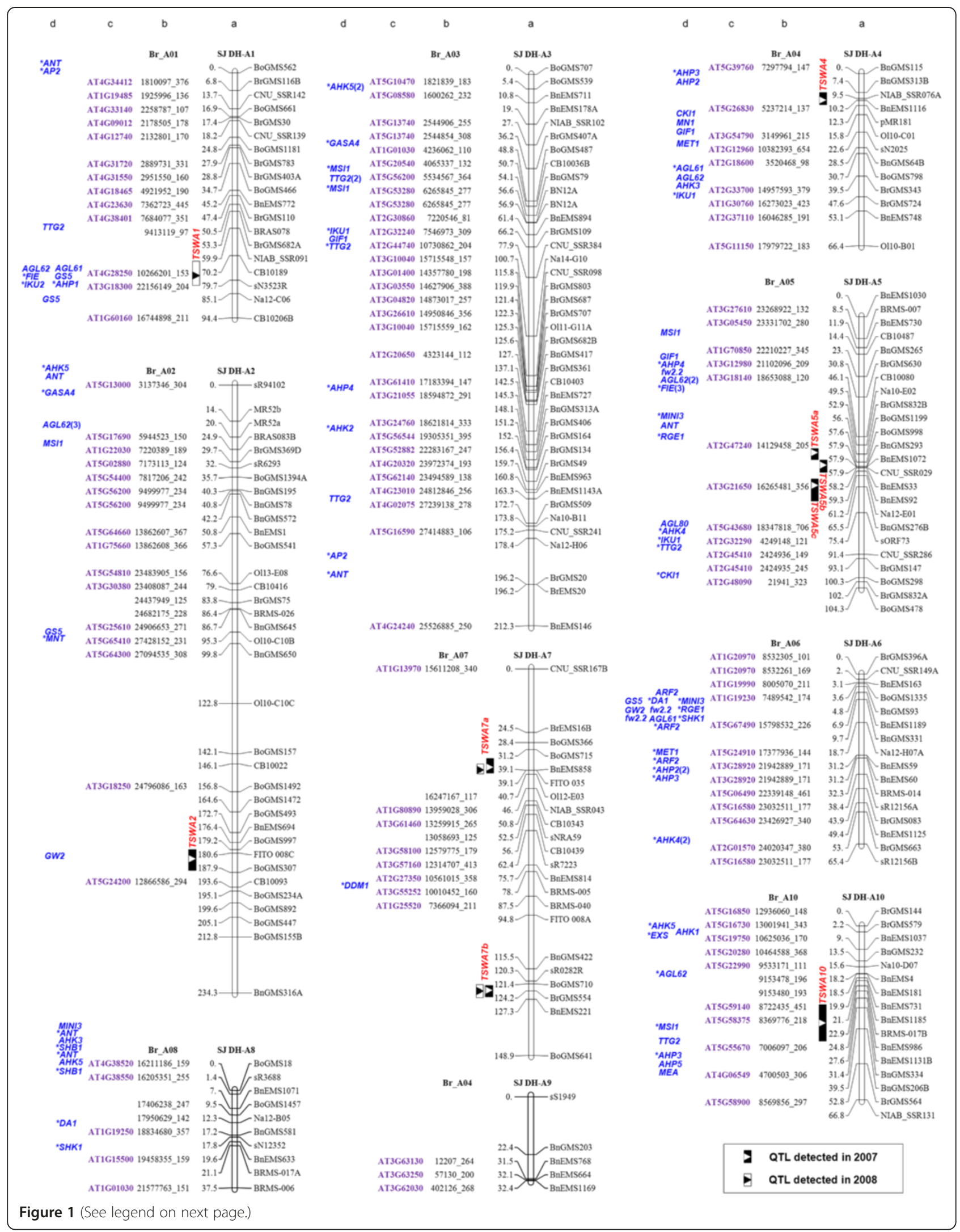


(See figure on previous page.)

Figure 1 Seed weight/size gene distribution map of B. napus (A genome). Column a presents the genetic linkage groups of the SJ DH population. The nomenclature of LGs follows the rules proposed by the Multinational Brassica Genome Project [37]. Each of the LGs is represented with a vertical bar with the locus position (in CM) on the left and SSR loci names on the right. The QTLs information (peak, interval and name) of TSW were on the left-hand of the LGs. Column $b$ lists the homologous colinear loci in B. rapa or B. oleracea. The numbers designate the physical position in B. rapa or B. oleracea chromosome with the length of amplification fragment. Column $\mathrm{C}$ is the Arabidopsis gene codes corresponding to the homologous loci. Column d lists the homologous genes of seed size or weight identified in B. rapa and B. oleracea. Genes that are in good fit into both Arabidopsis and B. rapa/B. oleracea physical positions are marked with asterisks. Numbers in brackets are the tandem repeat times of the tandem repeated genes.

were predicted with the software SEQUENCHER 4.1.2 (Gene Codes Corporation, Ann Arbor, MI, USA).

\section{Results}

Construction of the genetic linkage map and mapping of the QTLs for seed weight

Previously, we constructed a genetic map with 297 SSR markers for the SJ DH population [11]. In the present study, we expanded the SSR markers to 361 and reconstructed the map. In total, 397 SSR loci and 6 genespecific markers were located to the new genetic map with 19 linkage groups (LGs). The markers covered a genetic distance of 2,126.4 cM (the previous map was $2,011.1 \mathrm{cM}$ ) with an average distance of $5.36 \mathrm{cM}$ (the previous map was $6.15 \mathrm{cM}$ ) between markers (Figures 1, 2; Additional files 3, 4, 5 and 6: Figure S1-S4; Additional file 1: Table S1). The LGs corresponded to the 19 chromosomes of B. napus including A1-A10 (A genome) and C1-C9 (C genome) as determined by shared SSR markers in public genetic maps [37]. All SSR markers were evenly distributed across the whole genome of $B$. napus with 199 and 198 SSR loci on genome A and C, respectively (Table 1).

With the newly integrated map, the QTLs for TSW in the SJ DH population were re-scanned. A total of 12 QTLs of TSW were identified on 7 LGs (Additional file 7: Table S2), including three previously unidentified QTLs on LG C2 were detected in the year 2007, due to more molecular markers now available on the integrated map, which resulted in a higher density and better resolution in identification of subtle changes caused by genotypic effects. The distribution and effect of other QTLs, including two major QTLs (TSWA7a and TSWA7b) remained largely unchanged (Additional file 7: Table S2).

\section{Comparative mapping of $B$. napus and Arabidopsis} mediated with $B$. rapa and $B$. oleracea genome sequences With the primers (see Additional file 1: Table S1 for primer sequences) of the SSR markers mapped on the $B$. napus LGs, electronic-PCR (e-PCR) was performed using the genome sequence of $B$. rapa or $B$. oleracea as templates to obtain fragments amplified in respective genomes (amplicons). A computer program (e-PCRmap) was developed to operate the e-PCR process. In total,
945 amplified loci were obtained in the B. rapa and $B$. oleracea genomes with the primer sequences of 385 SSRs mapped on the SJ DH linkage map (Table 1 and Additional file 1: Table S1). From these analyses, 421 homologous colinear loci (amplicons that can be matched onto corresponding B. napus LGs) were identified (Table 1 ). Due to the highly colinearity between the Aand $\mathrm{C}$ - genome in B. napus, a SSR locus on a particular LG on $B$. napus may produce amplicons in both the $B$. rapa and $B$. oleracea genomes (Table 1; Additional files 3, 4, 5 and 6: Figure S1-S4).

Colinearity analysis between the $B$. napus LGs and the B. rapa/B. oleracea chromosomes showed the following three characteristics. First, some of B. napus LGs had high colinearity with the corresponding chromosomes of their progenitor species, $B$. rapa and B. oleracea, such as the LGs A1/C1, A2/C2, A3/C3, A4/C4 of B. napus that exhibited a sole colinear relationship with the chromosome A1, A2, A3, A4 in B. rapa and C1, C2, C3, C4 in B. oleracea, respectively (Figures 1 and 2; Additional file 3: Figure S1 and Additional file 4: Figure S2). Second, the LGs C6 and C7 of B. napus were colinear with the chromosome $\mathrm{C} 7$ and $\mathrm{C} 6$ of $B$. oleracea, respectively (Additional file 5: Figure S3). Such a corresponding relationship between LG C6 and chromosome C7 as well as LG C7 and chromosome C6 is likely resulted from the switched original labeling for $B$. napus linkage groups $[45,46]$, as pointed out by Panjabi et al. [33]. Third, some of $B$. napus LG had a complex colinear relationship with the progenitor species due to the translocations during the evolution of the tetraploid species. Such a complexity is characterized by that a single $B$. napus LG may have syntenic segments from several chromosomes from $B$. rapa and /or B. oleracea. For example, the B. rapa chromosome A7 (BrA7) was found to have colinear segments on B. napus LGs C6 and C7, while the BrA6 segments existed in both LGs A6 and C5, and the BoC8 in LGs A8, C8 and C9, respectively (Figures 1 and 2; Additional file 5: Figure S3 and Additional file 6: Figure S4). In addition, LG C5 contained a homologous segment of BoC05 at its upper part and a homologous segment of $\mathrm{BoC06}$ at its lower part (Figure 2). It was worth pointing out that B. napus LG C9 was largely colinear with BrA9, BoC8 and partly BoC3 simultaneously, rather than with 


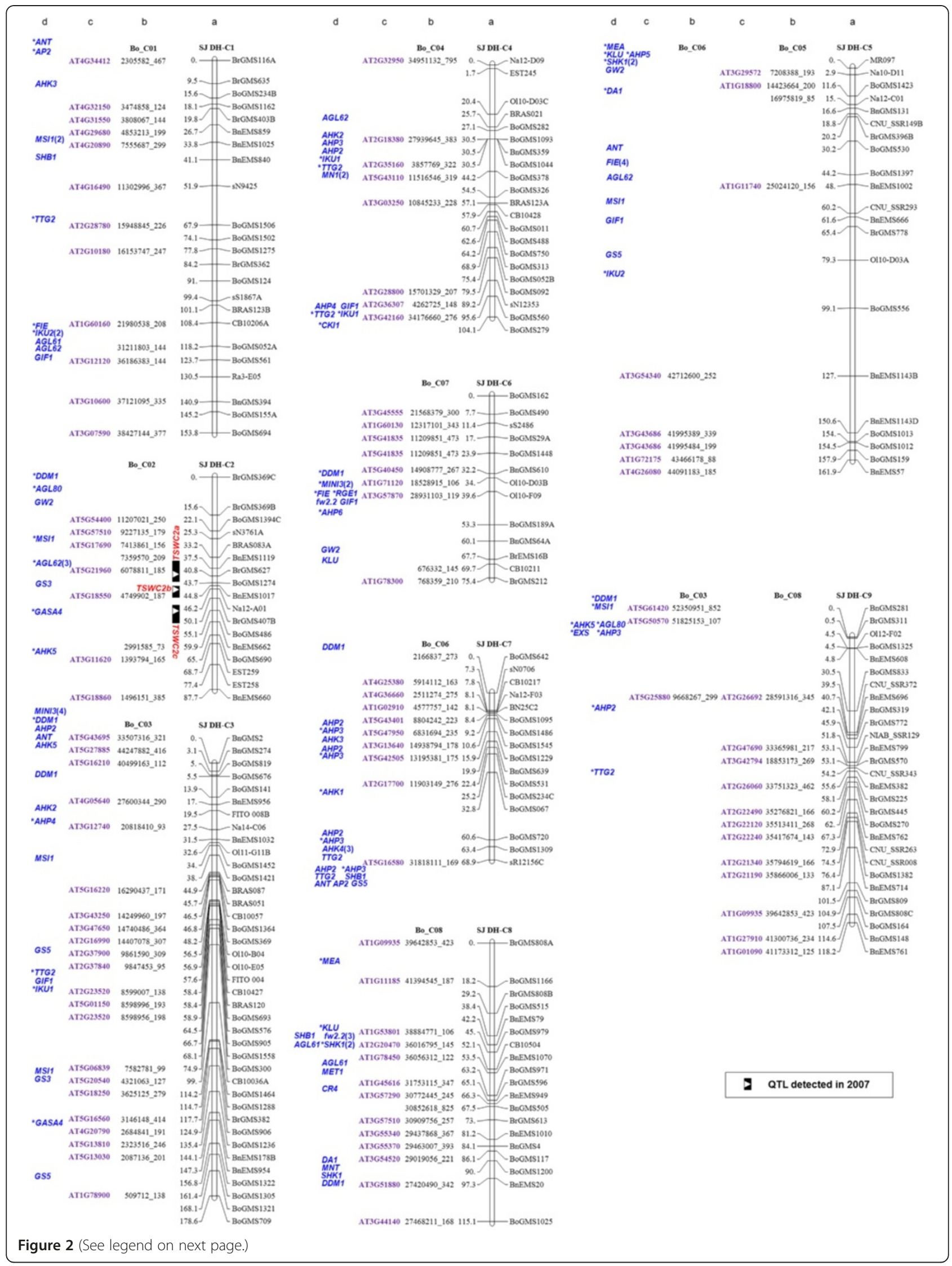


(See figure on previous page.)

Figure 2 Seed weight/size gene distribution map of B. napus (C genome). Column a presents the genetic linkage groups of the SJ DH population. The nomenclature of LGs follows the rules proposed by the Multinational Brassica Genome Project [37]. Each of the LGs is represented with a vertical bar with the locus position (in CM) on the left and SSR loci names on the right. The QTLs information (peak, interval and name) of TSW were on the left-hand of the LGs. Column b lists the homologous colinear loci in B. rapa or B. oleracea. The numbers designate the physical position in B. rapa or B. oleracea chromosome with the length of amplification fragment. Column $\mathrm{C}$ is the Arabidopsis gene codes corresponding to the homologous loci. Column d lists the homologous genes of seed size or weight identified in $B$. rapa and $B$. oleracea. Genes that are in good fit into both Arabidopsis and B. rapa/B. oleracea physical positions are marked with asterisks. Numbers in brackets are the tandem repeat times of the tandem repeated genes.

BoC9. The LG A9 was short and thus no corresponding colinear segments could be matched with it. The short LG A9 is likely due to the low polymorphism between the two parental lines and thus fewer markers available for this linkage group. Such a result was consistent with our previous analysis [11].

By BLASTn analysis [42] against the Arabidopsis genome sequences (TAIR10) [43], the 421 homologous colinear loci from the B. rapa and B. oleracea genomes were aligned onto 398 homologous loci in Arabidopsis, with 212 loci from B. rapa and 186 from B. oleracea, respectively (Tables 1 and 2; Additional files 3, 4, 5 and 6: Figure S1-S4). These homologous loci were evenly distributed in the $\mathrm{A}$ and $\mathrm{C}$ genomes of $B$. napus, with 208 loci in LG A1-A10, and 190 loci in LG C1-C9 (Table 2). There were 23 amplicons without matched

Table 1 Number of e-PCR amplicons and corresponding homologous colinear loci in B. rapa (A genome) and $B$. oleracea (C genome) for B. napus linkage groups

\begin{tabular}{|c|c|c|c|c|c|c|}
\hline \multicolumn{3}{|l|}{ B. napus } & \multicolumn{2}{|r|}{ B. rapa } & \multicolumn{2}{|r|}{ B. oleracea } \\
\hline LG & SSR loci & Amplicons in B. rapa and B. oleracea & Chr & Homologous colinear loci & Chr & Homologous colinear loci \\
\hline A1 & 19 & 35 & A1 & 14 & $\mathrm{C} 1$ & 13 \\
\hline $\mathrm{A} 2$ & 35 & 82 & A2 & 18 & $\mathrm{C} 2$ & 11 \\
\hline \multirow[t]{2}{*}{ A3 } & 39 & 71 & A3 & 30 & C3 & 15 \\
\hline & & & & & C6 & 7 \\
\hline A4 & 13 & 27 & A4 & 9 & C4 & 5 \\
\hline \multirow[t]{2}{*}{ A5 } & 24 & 69 & A5 & 12 & C5 & 4 \\
\hline & & & A6 & 6 & & \\
\hline \multirow[t]{2}{*}{ A6 } & 16 & 73 & A6 & 13 & C5 & 4 \\
\hline & & & & & C6 & 7 \\
\hline A7 & 22 & 31 & A7 & 10 & $\mathrm{C7}$ & 4 \\
\hline \multirow[t]{2}{*}{ A8 } & 10 & 23 & A8 & 7 & C3 & 3 \\
\hline & & & & & C8 & 3 \\
\hline A9 & 5 & 13 & A4 & 3 & C4 & 3 \\
\hline A10 & 16 & 40 & A10 & 12 & C9 & 9 \\
\hline Subtotal & 199 & 464 & & 134 & & 88 \\
\hline $\mathrm{C} 1$ & 24 & 69 & A1 & 10 & $\mathrm{Cl}$ & 13 \\
\hline$C 2$ & 17 & 46 & $\mathrm{~A} 2$ & 11 & $C 2$ & 9 \\
\hline$C 3$ & 39 & 67 & A3 & 18 & C3 & 23 \\
\hline C4 & 21 & 75 & A4 & 2 & C4 & 8 \\
\hline \multirow[t]{2}{*}{$\mathrm{C} 5$} & 21 & 45 & A1 & 3 & C5 & 4 \\
\hline & & & A6 & 6 & C6 & 5 \\
\hline C6 & 13 & 42 & A7 & 3 & $C 7$ & 9 \\
\hline C7 & 16 & 39 & A7 & 3 & C6 & 10 \\
\hline $\mathrm{C} 8$ & 19 & 46 & A9 & 11 & $\mathrm{C} 8$ & 14 \\
\hline \multirow[t]{2}{*}{ C9 } & 28 & 52 & A9 & 22 & C3 & 3 \\
\hline & & & & & $\mathrm{C} 8$ & 12 \\
\hline Subtotal & 198 & 481 & & 89 & & 110 \\
\hline Total & 397 & 945 & & 223 & & 198 \\
\hline
\end{tabular}


Table 2 Distribution of conserved Arabidopsis genomic blocks on B. napus genetic map based on homology analysis between B. napus and B. rapa/B. oleracea

\begin{tabular}{|c|c|c|c|c|c|c|c|c|c|}
\hline \multirow{2}{*}{$\frac{\text { B. napus }}{\text { LG }}$} & \multirow[t]{2}{*}{$\mathrm{Chr}^{\mathrm{a}}$} & \multicolumn{6}{|c|}{ Locus in A. thaliana } & \multirow[t]{2}{*}{$N^{b}$} & \multirow{2}{*}{$\begin{array}{l}\text { Conserved } \\
\text { block c }\end{array}$} \\
\hline & & AtC1 & AtC2 & AtC3 & AtC4 & AtC5 & Total & & \\
\hline \multirow[t]{2}{*}{ A1 } & $\mathrm{A} 1$ & 2 & & 1 & 10 & & 13 & 1 & 2 \\
\hline & $\mathrm{C} 1$ & 1 & & 1 & 11 & & 13 & & \\
\hline \multirow[t]{2}{*}{ A2 } & $A 2$ & 2 & & 2 & & 12 & 16 & 2 & 10 \\
\hline & $\mathrm{C} 2$ & 2 & & 2 & & 7 & 11 & & \\
\hline \multirow[t]{3}{*}{ A3 } & A3 & 1 & 4 & 9 & 4 & 12 & 30 & & 6 \\
\hline & $\mathrm{C} 3$ & 1 & 1 & 2 & 2 & 9 & 15 & & \\
\hline & $\mathrm{C} 6$ & 1 & 1 & & 3 & & 5 & 2 & \\
\hline \multirow[t]{2}{*}{ A4 } & A4 & 1 & 4 & 1 & & 3 & 9 & & 3 \\
\hline & C4 & 1 & 1 & 1 & & 2 & 5 & & \\
\hline \multirow[t]{3}{*}{ A5 } & A5 & 1 & 5 & 5 & & 1 & 12 & & 5 \\
\hline & A6 & 5 & & 1 & & & 6 & & \\
\hline & $C 5$ & 1 & & 3 & & & 4 & & \\
\hline \multirow[t]{3}{*}{$A 6$} & A6 & 4 & 1 & 2 & & 6 & 13 & & 5 \\
\hline & $\mathrm{C} 5$ & 2 & & & & 2 & 4 & & \\
\hline & C6 & & 2 & 1 & & 4 & 7 & & \\
\hline \multirow[t]{2}{*}{ A7 } & A7 & 3 & 1 & 4 & & & 8 & 2 & 3 \\
\hline & C7 & 2 & & 1 & & & 3 & 1 & \\
\hline \multirow[t]{3}{*}{ A8 } & A8 & 3 & & & 2 & & 5 & 2 & 3 \\
\hline & $\mathrm{C} 8$ & 3 & & & & & 3 & & \\
\hline & $\mathrm{C} 3$ & 2 & & & & & 2 & 1 & \\
\hline \multirow[t]{2}{*}{ A9 } & A4 & & & 3 & & & 3 & & 1 \\
\hline & C4 & & & 3 & & & 3 & & \\
\hline \multirow[t]{2}{*}{ A10 } & $\mathrm{A} 10$ & & & & 1 & 9 & 10 & 2 & 2 \\
\hline & C9 & 1 & & & & 7 & 8 & 1 & \\
\hline \multirow[t]{2}{*}{ Subtotal } & & 39 & 20 & 42 & 33 & 74 & B. rapa: 125 & 14 & 40 \\
\hline & & & & & & & B. oleracea: 83 & & \\
\hline \multirow[t]{2}{*}{$\overline{C 1}$} & $\mathrm{C} 1$ & 1 & 2 & 3 & 6 & & 12 & 1 & 3 \\
\hline & $\mathrm{A} 1$ & 1 & 1 & 4 & 4 & & 10 & & \\
\hline \multirow[t]{2}{*}{$\mathrm{C2}$} & $C 2$ & & & 1 & & 6 & 7 & 2 & 2 \\
\hline & $\mathrm{A} 2$ & 1 & 2 & & & 8 & 11 & & \\
\hline \multirow[t]{2}{*}{$\mathrm{C3}$} & $\mathrm{C} 3$ & 1 & 5 & 3 & 2 & 12 & 23 & & 7 \\
\hline & A3 & & 6 & 4 & 1 & 7 & 18 & & \\
\hline \multirow[t]{2}{*}{$\mathrm{C4}$} & C4 & & 5 & 2 & & 1 & 8 & & 3 \\
\hline & A4 & & & & 1 & 1 & 2 & & \\
\hline \multirow[t]{4}{*}{$\mathrm{C5}$} & $C 5$ & 2 & & 1 & & & 3 & 1 & 4 \\
\hline & C6 & 1 & & 3 & 1 & & 5 & & \\
\hline & A6 & 5 & & & & & 5 & 1 & \\
\hline & $\mathrm{A} 1$ & & & 3 & & & 3 & & \\
\hline C6 & C7 & 3 & & 2 & & 3 & 8 & 1 & 2 \\
\hline & A7 & 1 & & 1 & 1 & & 3 & & \\
\hline C7 & $\mathrm{C} 6$ & 1 & 1 & 1 & 2 & 4 & 9 & 1 & 2 \\
\hline & A7 & 1 & & 1 & & 1 & 3 & & \\
\hline C8 & $\mathrm{C} 8$ & 5 & 1 & 7 & & & 13 & 1 & 3 \\
\hline & A9 & 4 & 1 & 6 & & & 11 & & \\
\hline
\end{tabular}


Table 2 Distribution of conserved Arabidopsis genomic blocks on B. napus genetic map based on homology analysis between B. napus and B. rapa/B. oleracea (Continued)

\begin{tabular}{|c|c|c|c|c|c|c|c|c|c|}
\hline C9 & $\mathrm{C} 8$ & 3 & 8 & 1 & & & 12 & & 5 \\
\hline & A9 & 7 & 9 & 5 & & & 21 & 1 & \\
\hline & C3 & & & & & 3 & 3 & & \\
\hline \multirow[t]{2}{*}{ Subtotal } & & 37 & 41 & 48 & 18 & 46 & B. rapa: 87 & 9 & 31 \\
\hline & & & & & & & B. oleracea: & & \\
\hline Total & & 76 & 61 & 90 & 51 & 120 & 398 & 23 & 71 \\
\hline
\end{tabular}

${ }^{a}$ Chromosome of B. rapa (A-genome) or B. oleracea (C-genome).

${ }^{\mathrm{b}}$ Not determined.

c The number of unique blocks in a given B. napus LG. The conserved blocks were named according to Schranz et al. [5] and identified by the method described by Parkin et al. [30]. A conserved block contained a minimum of three mapped SSR loci with at least two homologous loci from one of the 24 defined Arabidopsis bocks [5] every $10 \mathrm{cM}$ in B. napus genetic map.

Arabidopsis homologous loci (Table 2 and Additional files 3, 4, 5 and 6: Figure S1-S4). In total, 71 Arabidopsis conserved blocks were resolved in the $B$. napus genome (Table 2 and Additional files 3, 4, 5 and 6: Figure S1-S4). The conserved blocks covered $1,411.3 \mathrm{cM}$ of $B$. napus genetic linkage map, accounting for $66.4 \%$ of total length of the genetic map (Additional file 8: Table S3).

Subsequently, a comparative map between Arabidopsis genome and the $B$. napus map based on SSR markers was constructed with the aid of $B$. rapa/B. oleracea genome sequences (Additional files 3, 4, 5 and 6: Figure S1-S4). In total, 385 SSR loci from B. napus exhibited synteny to Arabidopsis genes, but 114 SSR loci did not find any homologous regions in Arabidopsis (including 20 no amplification SSR loci). Altogether, 271 SSR loci on B. napus LGs were identified homologous to Arabidopsis (Additional files 3, 4, 5 and 6: Figure S1-S4; Additional file 1: Table S1).

The comparative map can be used to identify candidate genes of mapped QTLs

Previously, we identified several QTLs for seed weight and fatty acid content with the SJ-DH population, and cloned the candidate genes underlying the QTLs. Those QTLs include two QTLs for seed weight on A5 (TSWA5b and TSWA5c), one for oleic acid content on A5 (OLEA5), and two for linolenic acid content on A4 (LNAA4) and C4 (LNAC4), respectively [11,47]. To test whether the comparative map could be used to target the candidate genes for mapped QTLs, we searched for the candidate genes underlying the mapped QTLs. It was found that the F block on LG A5 contained a FAD2 gene for $O L E A 5$, the $\mathrm{H} / \mathrm{J}$ blocks on LG A4 and C4 harbored a FAD3 gene for $L N A A 4$ and $L N A C 4$, respectively (Figure $3 \mathrm{~A}$ and $3 \mathrm{C}$, column a). Similarly, the MINI3 gene for TSWA5b, TTG2 gene for TSWA5c could be predicted (Figure 3B, column a). Above predictions were exactly same as the previous analysis through homology cloning (Figure 3, column b) [11,47], demonstrating that the constructed comparative map can be effectively used in identification of candidate genes of mapped QTLs.

Locating the putative homologous genes for seed weight/size on $B$. napus genetic map by comparative mapping

Sequence information was collected for 43 genes involved in the regulation of seed/fruit size or weight previously reported in tomato, maize, rice and Arabidopsis (Table 3). The ORF sequences of the genes were used for BLAST analysis against the $B$. rapa and $B$. oleracea genome. In total, 286 corresponding genes/loci in the two species were obtained with 132 from $B$. rapa and 154 from $B$. oleracea, respectively (Table 3; Additional file 9: Table S4). Among the 286 loci, 244 corresponded to the 35 seed size genes from Arabidopsis and the rest of 42 to the 8 genes from other species (Table 4; Additional file 9: Table S4).

The copy numbers of the corresponding homologues in B. rapa and B. oleracea genomes varied. On average, one gene had 3 copies in the A genome and 3.6 copies in the $\mathrm{C}$ genome (Table 3 ). B. rapa and B. oleracea homologues for all genes were identified except for the gene $q S W 5 / G W 5$ from rice (Table 3).

Based on the colinear relationship between the A and $\mathrm{C}$ genome in three species (Table 1 ), the homologous genes were mapped onto the $B$. napus linkage map except the genes that currently are located only in the scaffolds of the B. rapa and B. oleracea genomes (Table 4; Figures 1 and 2, column d). Because of the fact that the LG C6 of B. napus was colinear with BoC7, and the LG $\mathrm{C} 7$ was colinear with BoC6, the homologous genes from BoC6 were placed on the LG C7 and BoC7 on LG C6, respectively (Figure 2). In total, 227 homologous genes of seed size/weight were finally positioned on the $B$. napus linkage map, which distributed on all LGs except A9 (Figures 1, 2; Table 4). Among the 185 Arabidopsis homologous genes mapped, 100 genes fell in conserved Arabidopsis genomic blocks and can be positioned exactly on the B. rapa/B. oleracea chromosomes 
A

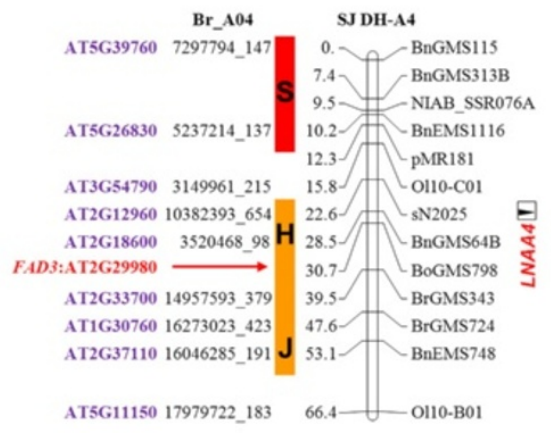

B

$$
\begin{aligned}
& \text { Br_A06 }
\end{aligned}
$$

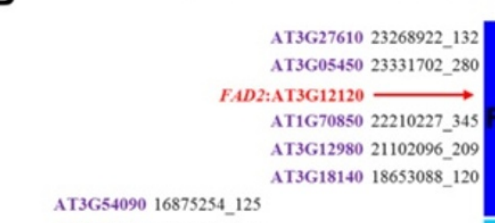

$$
\begin{aligned}
& \text { MINT3:AT1G55600 } \stackrel{ }{\stackrel{2477187 \_155}{\longrightarrow}} \\
& \text { AT1G74450 20995022_200 }
\end{aligned}
$$

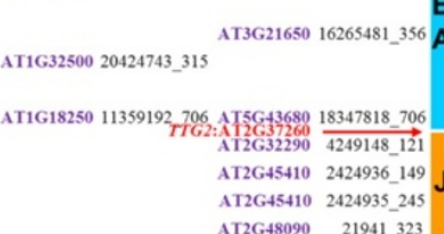$$
\text { AT1G28120 2477187_15 }
$$

\section{QTL detected by Fan et al. (2010) \\ QTL detected by Yang et al. (2012)}

C

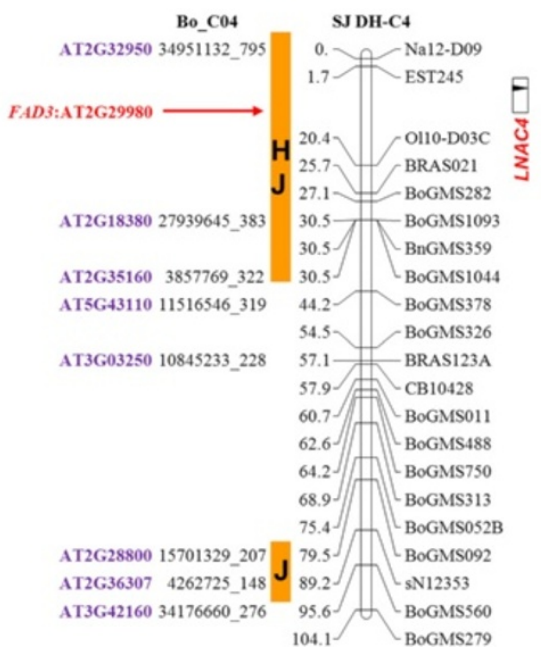

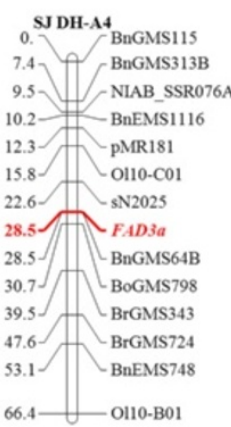

SJ DH-A5

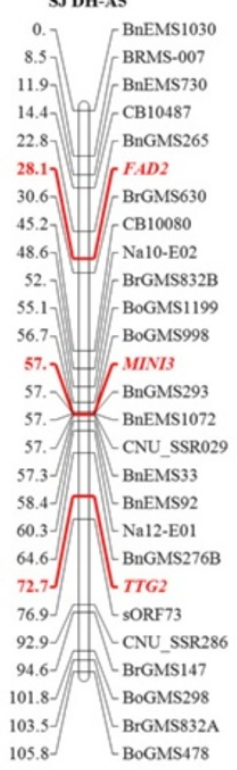

SJ DH-C4

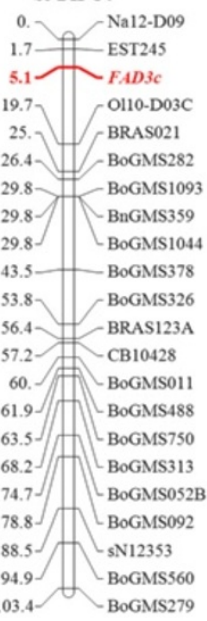

Figure $\mathbf{3}$ (See legend on next page.) 
(See figure on previous page.)

Figure 3 Candidate genes underlying the QTLs identified through comparative mapping. Candidate genes for mapped QTLs on LGS A4 (A), A5 (B) and C4 (C) are predicted with conserved Arabidopsis blocks mapped on B. napus map. Column a illustrates the prediction of the candidate genes. Previously mapped QTLs $[11,47]$ are indicated on the right-hand side of the respective LG and the conserved Arabidopsis blocks (color bars with their names (letters) inside) on the left. The homologous loci in B. rapa/B. oleracea and Arabidopsis are listed next to the conserved blocks. Red arrows indicate the positions of the predicted candidate genes. Column b presents LGs A4 (A), A5 (B) and C4 (C) with newly added gene-specific markers.

(Figures 1 and 2, genes with asterisk). There were 20 homologous genes with tandem repeats (TR), among which three were from the homologous genes in crop species and 17 from Arabidopsis. Seventeen TRs genes were anchored onto the linkage map and the other three located in scaffolds of $B$. rapa/B. oleracea (Figures 1 and 2).

\section{Identification of the candidate genes underlying QTLs for seed weight in $B$. napus}

The seed size/weight gene distribution map was used to identify candidate genes for the TSW QTLs by aligning the seed weight genes with the TSW QTL loci on the $B$. napus genetic map (Figures 1 and 2). On LG A1, TTG2 and GS5 were the nearest genes on each side of TSWA1 (Figure 1 and Additional file 9: Table S4); GW2 was located at the same position of TSWA2 (Figure 1 and Additional file 9: Table S4); CKI1 and MN1 were nearby the peak of TSWA4 (Figure 1 and Additional file 9: Table S4). MINI3 and FIE were located nearby previously mapped TSWA5a and TSWA5b. The MINI3 gene was located in the confidence interval of TSWA $5 \mathrm{~b}$, consistent to our previous results [11], while the FIE gene fell into the confidence interval of TSWA5a (Figure 1 and Additional file 9: Table S4). Three genes, AHP3, AHP5 and $M E A$, were located in the same confidence interval of TSWA10 (Figure 1 and Additional file 9: Table S4). In addition, AGL62, GS3 and GASA4 were located on the peaks or in the confidence intervals of three newly identified QTLs, TSWC $2 a$, TSWC $2 b$ and TSWC2c, respectively (Figure 2 and Additional file 9: Table S4). Together, above analysis clearly pinpointed the potential target

Table 3 Genes involved in seed size/weight regulation and their homologues in B. rapa and B. oleracea

\begin{tabular}{|c|c|c|c|c|c|c|c|c|c|}
\hline \multirow[t]{2}{*}{ Gene } & \multirow[t]{2}{*}{ Species } & \multirow[t]{2}{*}{ Ref. } & \multicolumn{2}{|c|}{ Copy number } & \multirow[t]{2}{*}{ Gene } & \multirow[t]{2}{*}{ Species } & \multirow[t]{2}{*}{ Ref. } & \multicolumn{2}{|c|}{ Copy number } \\
\hline & & & B. rapa & B. oleracea & & & & B. rapa & B. oleracea \\
\hline fw2.2 & Tomato & [48] & 6 & 4 & AGL61 & Arabidopsis & [49] & 5 & 4 \\
\hline MN1 & Maize & {$[50]$} & 1 & 2 & AGL62 & Arabidopsis & [51] & 8 & 7 \\
\hline CR4 & Maize & {$[52]$} & 1 & 1 & AHP1 & Arabidopsis & [53] & 1 & 1 \\
\hline GW2 & Rice & [54] & 2 & 3 & AHP2 & Arabidopsis & [53] & 4 & 8 \\
\hline GS3 & Rice & [55] & 0 & 2 & AHP3 & Arabidopsis & [53] & 4 & 7 \\
\hline GW5 & Rice & {$[56,57]$} & 0 & 0 & AHP4 & Arabidopsis & [53] & 3 & 2 \\
\hline GS5 & Rice & [18] & 5 & 5 & AHP5 & Arabidopsis & [53] & 1 & 1 \\
\hline GIF1 & Rice & {$[58]$} & 3 & 7 & AHP6 & Arabidopsis & [53] & 0 & 1 \\
\hline MINI3 & Arabidopsis & [59] & 4 & 7 & ANT & Arabidopsis & [60] & 6 & 5 \\
\hline$A P 2$ & Arabidopsis & [61] & 3 & 2 & CKI1 & Arabidopsis & {$[62]$} & 2 & 2 \\
\hline IKU1 & Arabidopsis & [63] & 3 & 3 & DDM1 & Arabidopsis & [64] & 2 & 8 \\
\hline IKU2 & Arabidopsis & [59] & 1 & 3 & EMS1 & Arabidopsis & [65] & 2 & 1 \\
\hline SHB1 & Arabidopsis & {$[66]$} & 4 & 6 & FIS3 & Arabidopsis & [67] & 5 & 6 \\
\hline ARF2 & Arabidopsis & [68] & 8 & 4 & FIS2 & Arabidopsis & [69] & 0 & 1 \\
\hline TTG2 & Arabidopsis & [70] & 7 & 8 & GASA4 & Arabidopsis & [71] & 2 & 2 \\
\hline AHK1 & Arabidopsis & {$[72]$} & 1 & 1 & FIS1 & Arabidopsis & [67] & 1 & 2 \\
\hline AHK2 & Arabidopsis & [72] & 2 & 2 & MET1 & Arabidopsis & [73] & 3 & 3 \\
\hline AHK3 & Arabidopsis & [72] & 3 & 4 & MSI1 & Arabidopsis & [67] & 6 & 7 \\
\hline AHK4 & Arabidopsis & [72] & 4 & 3 & RGE1 & Arabidopsis & [74] & 2 & 2 \\
\hline AHK5 & Arabidopsis & [72] & 6 & 5 & SHK1 & Arabidopsis & [75] & 4 & 5 \\
\hline$K L U$ & Arabidopsis & [9] & 3 & 3 & $D A 1$ & Arabidopsis & [76] & 2 & 2 \\
\hline AGL80 & Arabidopsis & [77] & 2 & 2 & & & & & \\
\hline Total & & & & & 43 & & & 132 & 154 \\
\hline
\end{tabular}


Table 4 Number of homologous genes for seed size/weight in B. napus

\begin{tabular}{lllllll}
\hline LG & \multicolumn{2}{c}{ Gene origin } & LG & \multicolumn{2}{c}{ Gene origin } \\
\cline { 2 - 4 } \cline { 5 - 6 } & Arabidopsis & $\begin{array}{l}\text { Other } \\
\text { species }\end{array}$ & & & Arabidopsis & $\begin{array}{l}\text { Other } \\
\text { species }\end{array}$ \\
\hline A1 & 8 & 2 & C1 & 12 & 1 \\
\hline A2 & 8 & 2 & C2 & 8 & 2 \\
\hline A3 & 14 & 1 & C3 & 16 & 4 \\
\hline A4 & 8 & 2 & C4 & 10 & 3 \\
\hline A5 & 15 & 2 & C5 & 14 & 3 \\
\hline A6 & 14 & 4 & C6 (BoC7) & a & 7 & 3 \\
\hline A7 & 1 & 0 & C7 (BoC6) & b & 19 & 1 \\
\hline A8 & 9 & 0 & C8 & 12 & 4 \\
\hline A9 & 14 & 4 & C9 & 8 & 0 \\
\hline A10 & 10 & 0 & & & 3 \\
\hline Scaffold & 13 & 1 & Scaffold & 24 & \\
\hline Total & $\mathbf{1 1 4}$ & $\mathbf{1 8}$ & Total & $\mathbf{1 3 0}$ & $\mathbf{2 4}$ \\
\hline
\end{tabular}

${ }^{a}$ The homologous gene number was calculated based on the distribution on BoC7 because the LG C6 of $B$. napus has a high colinearity with BoC7.

${ }^{b}$ The homologous gene number was calculated based on the distribution on BoC6 because the LG C7 of $B$. napus has a high colinearity with BoC6.

genes for the mapped QTLs, providing valuable clues for a further comparison of sequence differences between two parental lines.

However, for the two major QTLs, TSWA7a and $T S W A 7 b$, no known information about candidate genes could be inferred from the map, suggesting that those QTLs may be unique in B. napus or $B$. rapa genome. The only one seed size/weight gene, DDM1, on LG A7 was far away from these two QTLs, thus unlikely the candidate gene for the two QTLs (Figure 1).

\section{Molecular cloning of BnAP2 gene and development of gene-specific marker}

The Arabidopsis AP2 (AtAP2, AT4G36920) gene has been reported to affect seed size [61]. AtAP2 was located on the U-block of LG A1 in the comparative map (Figure 4A). Although no mapped QTL is matched to the predicted AP2 gene, we reasoned that it is possible to find polymorphism of the locus between the two parental lines. To test this hypothesis, we set out to clone the homologous gene of AtAP2 in B. napus. We searched for the homologous sequences in $B$. rapa genome with BLASTn by using AT4G36920 as a query. Primers were designed based on the retrieved sequence of $B r A P 2$ gene in $B$. rapa and the $B n A P 2$ gene was cloned in the two parental lines of the SJ DH population, respectively (Figure $4 \mathrm{~B}$ and $4 \mathrm{C}$; Additional file 10: Figure S5). Sequence analysis showed that the allele in SW Hickory contained a 290bp insertion compared to JA177 (Figure 4C and Additional file 10: Figure S5). A BnAP2 gene-specific marker was developed based on the polymorphism between the two parents and used to map the gene in the SJ-DH population again. Genetic linkage analysis eventually mapped the $B n A P 2$ genespecific marker onto the $U$ block on LG A1, consistent with the predicted result on the comparative map (Figure 4A).

\section{Discussion}

In this study, we developed a procedure for comparative mapping between B. napus and Arabidopsis with SSR markers with the aid of $B$. rapa and $B$. oleracea genome sequences. To the best of our knowledge, this is the first report to construct a comparative map among Arabidopsis and three Brassica species with a SSR-based genetic map (Additional files 3, 4, 5 and 6: Figure S1-S4; Additional file 1: Table S1). The SSR markers have been widely used as a preferable type of molecular marker in genetic mapping in Brassica species. However, it was difficult to use a SSR map for comparative mapping with Arabidopsis directly. First, individual SSR primer pairs only have limited sequence information, which renders a direct alignment with Arabidopsis genome ineffective. For example, in a study to construct a mainly SSR-based integrated map in B. napus, Wang et al. [32] found that $<2 \%$ of the primer pairs could identify homologous regions to Arabidopsis, of which only 50\% agreed with those identified using the corresponding SSR clone sequences. Second, high homology between the A and C genomes often results in multiple polymorphic loci in $B$. napus for a single Arabidopsis gene, which further complicates the comparative analysis between $B$. napus and Arabidopsis. In this study, we circumvented the two difficulties by making use of recently released genomic sequences of $B$. rapa and B. oleracea. Through anchoring the SSR loci on B. napus LGs to the B. rapa/ $B$. oleracea genome by e-PCR, we were able to match the B. napus SSR loci with their Arabidopsis homologues, thus making such a comparative mapping feasible. By overcoming the difficulties in comparative mapping using a SSR-based genetic map of B. napus and Arabidopsis genomic sequences, this procedure thus proved a novel idea for a comprehensive comparison among Arabidopsis, B. napus and its two progenitor species, B. rapa and B. oleracea.

To make use of the information derived the SSR loci as much as possible, a less stringent E-value was initially used in this study to identify more putative homologous loci. As indicated by Lukens et al. [29], a less stringent cutoff could result in more non-specific region of homology. However, since our major purpose in this study was to establish colinear relationships between $B$. napus and Arabidopsis through the conserved blocks, such non-specific homology regions in the initial screening will be re-examined. With the criterion for identification 
A

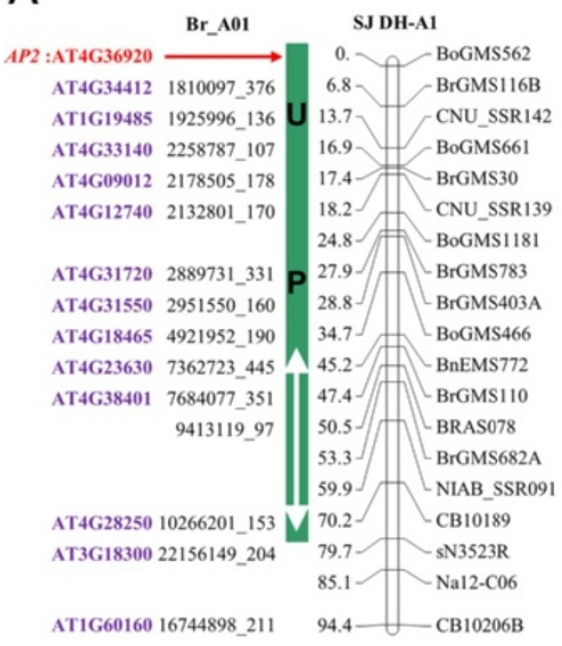
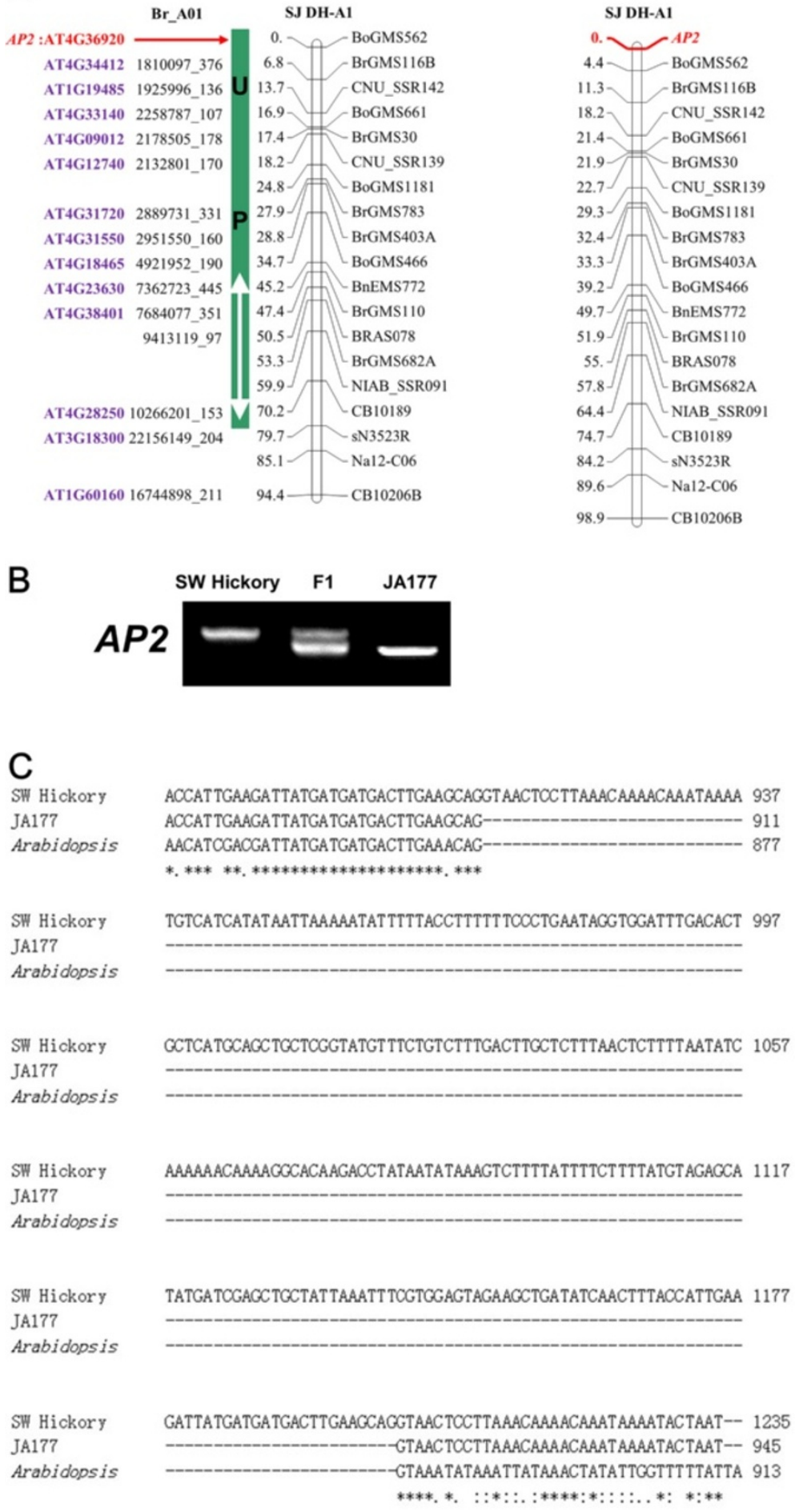

Figure 4 Cloning of the BnAP2 gene with the aid of the comparative map. A) Localization of the BnAP2 gene and its allele-specific marker on LG A1. The red arrow on the left panel marks the AP2 position as predicted in conserved block $U$. The right panel is a reconstructed $L G A 1$ with the BnAP2 allele-specific marker. B) PCR products amplified from the parental lines and their $F_{1}$. The PCR products are separated by electrophoresis in 1.0\% agarose gels and stained with ethidium bromide. C) Sequence difference of the BnAP2 gene sequences between the two parental lines. There is a 290bp insertion in SW Hickory. 
of conserved blocks, such non-specific loci will not affect the determination of the conserved blocks. This is evident through the data listed in Additional file 1: Table S1, in which about $66 \%$ of the loci under the less stringent (E-value $>1 \mathrm{E}-05)$ cutoff eventually were linked to a perspective block, indicating that some weak but biologically relevant sequence relationships could be revealed with such a procedure, which reduces the loss of valuable information from the SSR loci on the B. napus map.

The establishment of such a comparative map offers an effective way to transfer the gene information from model plant Arabidopsis to B. napus, an amphidiploid crop species, as demonstrated by mapping the seed size/ weight genes on the $B$. napus genetic map (Figures 1 and 2). Furthermore, we identified candidate genes for eight TSW QTLs through the mapping (Figures 1 and 2; Additional files 3, 4, 5 and 6: Figure S1-S4). Together, the seed distribution map and the identified candidate genes for mapped TSW QTLs provide valuable information about the genetic control of seed weight in $B$. napus. Although such a list of seed size/weight genes could be further expanded by including other genes related to the process of seed development, our results do exemplify the universal usefulness of such an approach. A flow diagram for the process is presented in Additional file 11: Figure S6.

Mapping of the seed weight related genes and the candidate genes for TSW QTLs could accelerate the molecular cloning and functional characterization of the QTLs. As shown in Figure 3, the prediction of the candidate genes for several mapped QTLs is accurate. Such a process will allow us to isolate the potential candidate genes for a particular QTL by homologous cloning strategy rather than tedious and time-consuming traditional map-based cloning procedure. On the other hand, by cloning some of predicted potential candidate genes that were even not located in the genetic map, for example AP2 in this study, it is possible to uncover the polymorphic alleles in two parental lines without QTL mapping information (Figure 4). By doing so, we were able to develop an allele-specific marker for one of locus of the AP2 gene in B. napus and place the marker on the corresponding LG (Figure 4). There are three and two copies of the AtAP2 homologues identified in B. rapa (including one copy located on a scaffold) and B. oleracea, respectively (Table 3; Additional file 9: Table S4). Consistently, there are four copies mapped on LGs A1, $\mathrm{A} 3, \mathrm{C} 1$ and $\mathrm{C} 7$ of the B. napus genetic map, respectively (Figures 1 and 2). Although the exact molecular significance of the insertion in the cloned BnAP2 allele of SW Hickory is yet to be established, identification of the polymorphic locus between the two parental lines lays foundation for further functional characterization of all the $A P 2$ alleles in the $B$. napus genome.
The seed weight genetic map revealed the complexity of the genetic control of seed weight in amphidiploid rapeseed. For example, a single TSW QTL may have one or multiple candidate gene(s), such as TSWA2 (with only one gene, GW2, located) and TSWA1 (with 6 genes located) (Figure 1 and Additional file 9: Table S4). Mapping of these candidate genes could apparently narrow down the range of the potential target genes. Of course, even though potential candidate genes are mapped to a locus, this does not imply that they control the trait. The QTL may result from variation in other novel genes which have not been studied in model systems.

It is interesting to notice that some genes showing major effects on seed size/weight in rice and Arabidopsis, such as GS3, GS5, GW2 and MINI3, TTG2, ARF2, IKU2, were located on the minor QTLs regions, or even not in the confidence intervals of previously mapped TSW QTLs (Figures 1 and 2; Additional file 9: Table S4). In addition, no homologue of $q S W 5 / G W 5$, an important rice seed size gene $[56,57]$ could be identified in both the Aand $\mathrm{C}$ - genome (Table 3). A more comprehensive evaluation of $B$. napus germplasm is needed to understand whether these genes may exhibit different effects on the studied trait in various species. On the other hand, no candidate genes for two previously mapped major QTLs, $T S W A 7 a$ and TSWA7b were identified, suggesting that the two QTLs may represent novel determinants for seed weight in amphidiploid B. napus.

\section{Conclusions}

We developed a procedure for comparative mapping between $B$. napus and $A$. thaliana with SSR markers with the aid of $B$. rapa and $B$. oleracea genome sequences. Such a SSR-based genetic map can be used to pinpoint the candidate genes for QTLs important in crop improvement. The procedure may also find wide applicability in Brassicaceae and other crop species, as candidate genes for QTLs in other pathways could be identified through a similar approach.

\section{Additional files}

\footnotetext{
Additional file 1: Table S1. Genetic linkage groups of B. napus, SSR marker primer sequences, amplification copy numbers, amplicon position in B. rapa/B. oleracea and homologous colinear locus in Arabidopsis.

Additional file 2: The Perl script of e-PCRmap computer program.

Additional file 3: Figure S1. Comparative map of $B$. napus with $A$ thaliana (LGs $A 1, A 2, C 1$ and $C 2$ ). Column a presents the genetic linkage groups (LGS) of the SJ DH population. LGs are represented by vertical bars with the loci position (in CM) indicated on the left and SSR loci names on the right. Column $b$ and $c$ list the homologous colinear loci in $B$. rapa and $B$. oleracea, respectively. The number means the physical position in B. rapa or B. oleracea chromosome with the length of amplification fragment. Column $\mathrm{d}$ and e are Arabidopsis gene codes corresponding to the homologous loci. Column $\mathrm{f}$ is the Arabidopsis conserved blocks identified in B. napus, which is nomenclatured
} 
according to Schranz et al. [5] and colored differently based on the A. thaliana (At) chromosome positions defined by Parkin et al. [30]. Inversions in the linkage groups relative to Arabidopsis are indicated by arrows. Column $g$ lists the homologous genes of seed size or weight in B. rapa and B. oleracea. Genes with asterisk indicate that they are in good fit into both Arabidopsis and B. rapa/B. oleracea physical positions, and genes with brackets are the tandem repeats (TR) of homologous genes with the tandem repeat times in brackets.

Additional file 4: Figure S2. Comparative map of $B$. napus with A. thaliana (LGs A3, A4 C3 and C4).

Additional file 5: Figure S3. Comparative map of $B$. napus with A. thaliana (LGs A5-A7 and C5-C7).

Additional file 6: Figure S4. Comparative map of $B$. napus with A. thaliana (LGS A8-A10, C8 and C9).

Additional file 7: Table S2. QTLS for TSW in the SJ DH population detected previously and re-scanned in the present study.

Additional file 8: Table S3. Distribution of conserved Arabidopsis genomic blocks in B. napus genome.

Additional file 9: Table S4. Homologous genes of seed size/weight in B. rapa and B. oleracea chromosomes and scaffolds,

Additional file 10: Figure S5. Comparative analysis of $A P 2$ genomic sequences of SW Hickory and JA177 with Arabidopsis.

Additional file 11: Figure S6. A flow diagram for construction of a comparative map between $B$. napus with Arabidopsis based on the $B$. rapa and $B$. oleracea genome sequences, and for prediction of candidate genes for QTLs mapped on the map.

\section{Competing interests}

The authors declare that they have no competing interests.

\section{Authors' contributions}

YZ and GC conceived the research. GC, YY, QY, ZZ, HC, JW and CF performed genetic mapping and collected the seed gene data. $Y Y$ wrote the computer program and assisted in bioinformatic analysis. GC and YY performed the comparative mapping. GC carried out the molecular cloning. GC and YZ wrote the paper. All authors read and approved the final manuscript.

\section{Acknowledgements}

We thank Professor Edgar Cahoon at Center for Plant Science Innovation, University of Nebraska-Lincoln, USA and Dr. Muhammad Younas at National Key Laboratory of Crop Genetic Improvement, Huazhong Agricultural University, China, for critical reading of the manuscript. We are grateful to two anonymous reviewers for their valuable comments and suggestions on the manuscript. The work is financially supported by the funding from Ministry of Science and Technology of China (Grant nos. 2006CB101604, 2006AA10A113 and 2011AA10A104), Ministry of Agriculture of China (nycytx-00503), National Natural Science Foundation of China (31171188) and Huazhong Agricultural University (STSIF 2010YB05).

Received: 22 July 2012 Accepted: 30 November 2012

Published: 6 December 2012

\section{References}

1. $U$ N: Genome analysis in Brassica with special reference to the experimental formation of $B$. napus and peculiar mode of fertilization. Jpn J Bot 1935, 7:389-452.

2. Lan TH, DelMonte TA, Reischmann KP, Hyman J, Kowalski SP, McFerson J: An EST-enriched comparative map of Brassica oleracea and Arabidopsis thaliana. Genome Res 2000, 10:776-788.

3. O'Neill CM, Bancroft I: Comparative physical mapping of segments of the genome of Brassica oleracea var. alboglabra that are homoeologous to sequenced regions of chromosomes 4 and 5 of Arabidopsis thaliana. Plant J 2000, 23:233-243.

4. Park JY, Koo DH, Hong CP, Lee SJ, Jeon JW, Lee SH, Yun PY, Park BS, Kim HR, Bang JW, Plaha P, Bancroft I, Lim YP: Physical mapping and microsynteny of Brassica rapa ssp. pekinensis genome corresponding to a $222 \mathrm{kbp}$ gene-rich region of Arabidopsis chromosome 4 and partially duplicated on chromosome 5. Mol Genet Genomics 2005, 274:579-588.
5. Schranz ME, Martin AL, Mitchell-Olds T: The ABC's of comparative genomics in the Brassicaceae: building blocks of crucifer genomes. TRENDS in Plant Science 2006, 11:535-542.

6. The Brassica rapa Genome Sequencing Project Consortium: The genome of the mesopolyploid crop species Brassica rapa. Nat Genet 2011, 43:1035-1039.

7. Morgan CL, Arthur AE, Rawsthorne S: Influence of testa colour and seed size on storage product composition in Brassica juncea. Plant Varieties Seeds 1998, 11:73-81.

8. Lionneton E, Aubert $\mathrm{G}$, Ochatt $\mathrm{S}$, Merah $\mathrm{O}$ : Genetic analysis of agronomic and quality traits in mustard (Brassica juncea). Theor Appl Genet 2004, 109:792-799.

9. Adamskia NM, Anastasioub E, Erikssona S, O'Neillc CM, Lenharda M: Local maternal control of seed size by KLUH/CYP78A5-dependent growth signaling. Proc Natl Acad Sci USA 2009, 106:20115-20120.

10. Teng W, Han Y, Du Y, Sun D, Zhang Z, Qiu L, Sun G, Li W: QTL analyses of seed weight during the development of soybean (Glycine max L. Merr.). Heredity 2009, 102:372-380.

11. Fan CC, Cai GQ, Qing J, Li QY, Yang MG, Wu J, Fu TD, Liu KD, Zhou YM: Mapping of quantitative trait loci and development of allele-specific markers for seed weight in Brassica napus. Theor Appl Genet 2010, 121:1289-1301.

12. Ramya P, Chaubal A, Kulkarni K, Gupta L, Kadoo N, Dhaliwal HS, Chhuneja P, Lagu M, Gupta V: QTL mapping of 1000-kernel weight, kernel length, and kernel width in bread wheat (Triticum aestivum L.). J Appl Genet 2010, 51:421-429.

13. Tsilo T, Hareland G, Simsek S, Chao S, Anderson J: Genome mapping of kernel characteristics in hard red spring wheat breeding lines. Theor Appl Genet 2010, 121:717-730

14. Zhang LW, Yang GS, Liu PW, Hong DF, Li SP, He QB: Genetic and correlation analysis of silique-traits in Brassica napus L. by quantitative trait locus mapping. Theor Appl Genet 2011, 122:21-31.

15. Sundaresan V: Control of seed size in plants. Proc Natl Acad Sci USA 2005, 102:17887-17888.

16. Sun XD, Shantharaj D, Kang XJ, Ni M: Transcriptional and hormonal signaling control of Arabidopsis seed development. Current Opinion in Plant Biology 2010, 13:611-620.

17. Xing YZ, Zhang QF: Genetic and molecular bases of rice yield. Annu Rev Plant Biol 2010, 61:421-442.

18. Li YB, Fan CC, Xing YZ, Jiang Y, Luo L, Sun L, Shao D, Xu CG, Li XH, Xiao JH, He YQ, Zhang QF: Natural variation in GS5 plays an important role in regulating grain size and yield in rice. Nat Genet 2011, 43:1266-1269.

19. Liu DF, Liu HL: Studies on genetic variation of quantitical traits in Brassica napus L. Acta Genet Sin 1987, 14:31-36.

20. Qi CK, Gai JY, Fu SZ, Pu HM, Zhang JF, Chen XJ, Gao JQ: Analysis of genetic system of 1,000 seed weight in Brassica napus L. Acta Genet Sin 2004, 30:1274-1277.

21. Shi JQ, Li RY, Qiu D, Jiang CC, Long Y, Morgan C, Bancroft I, Zhao JY, Meng $\mathrm{JL}$ : Unraveling the complex trait of crop yield with quantitative trait loci mapping in Brassica napus. Genetics 2009, 182:851-861.

22. Quijada PA, Udall JA, Lambert B, Osborn TC: Quantitative trait analysis of seed yield and other complex traits in hybrid spring oilseed rape (Brassica napus L.): 1. Identification of genomic regions from winter germplasm. Theor Appl Genet 2006, 113:549-561.

23. Udall JA, Quijada PA, Lambert B, Osborn TC: Quantitative trait analysis of seed yield and other complex traits in hybrid spring rapeseed (Brassica napus L.): 2. Identification of alleles from unadapted germplasm. Theor Appl Genet 2006, 113:597-609.

24. Basunanda P, Radoev M, Ecke W, Friedt W, Becker HC, Snowdon RJ: Comparative mapping of quantitative trait loci involved in heterosis for seedling and yield traits in oilseed rape (Brassica napus L.). Theor Appl Genet 2010, 120:271-281

25. Arabidopsis Genome Initiative: Analysis of the genome sequence of the flowering plant Arabidopsis thaliana. Nature 2000, 408:796-815.

26. Kowalski SP, Lan TH, Feldmann KA, Paterson AH: Comparative mapping of Arabidopsis thaliana and Brassica oleracea chromosomes reveals islands of conserved organization. Genetics 1994, 138:499-510.

27. Lagercrantz U: Comparative mapping between Arabidopsis thaliana and Brassica nigra indicates that Brassica genomes have evolved through extensive genome replication accompanied by chromosome fusions and frequent rearrangements. Genetics 1998, 150:1217-1228. 
28. Ryder CD, Smith LB, Teakle GR, King GJ: Contrasting genome organisation: two regions of the Brassica oleracea genome compared with collinear regions of the Arabidopsis thaliana genome. Genome 2001, 44:808-817.

29. Lukens L, Zou F, Lydiate D, Parkin IAP, Osborn T: Comparison of a Brassica oleracea genetic map with the genome of Arabidopsis thaliana. Genetics 2003, 164:359-372.

30. Parkin IAP, Gulden SM, Sharpe AG, Lukens L, Trick M, Osborn TC, Lydiate DJ: Segmental structure of the Brassica napus genome based on comparative analysis with Arabidopsis thaliana. Genetics 2005, 171:765-781.

31. Kaczmarek M, Koczyk G, Ziolkowski PA, Danuta B-S, Sadowski J, Kaczmarek M, Koczyk G, Ziolkowski PA, Babula-Skowronska D, Sadowski J: Comparative analysis of the Brassica oleracea genetic map and the Arabidopsis thaliana genome. Genome 2009, 52:620-633.

32. Wang J, Lydiate DJ, Parkin IAP, Falentin C, Delourme R, Carion PWC, King GJ: Integration of linkage maps for the Amphidiploid Brassica napus and comparative mapping with Arabidopsis and Brassica rapa. BMC Genomics 2011, 12:101-122.

33. Panjabi P, Jagannath A, Bisht NC, Padmaja KL, Sharma S, Gupta V: Comparative mapping of Brassica juncea and Arabidopsis thaliana using Intron Polymorphism (IP) markers: homoeologous relationships, diversification and evolution of the $\mathrm{A}, \mathrm{B}$ and $\mathrm{C}$ Brassica genomes. BMC Genomics 2008, 9:113-131.

34. Zhao JY, Huang JX, Chen F, Xu F, Ni XY, Xu HM, Wang YL, Jiang CC, Wang $H$, Xu AX, Huang RZ, Li DR, Meng JL: Molecular mapping of Arabidopsis thaliana lipid-related orthologous genes in Brassica napus. Theor Appl Genet 2012, 124:407-421.

35. Cheung F, Trick M, Drou N, Lim YP, Park J-Y, Kwon S-J, Kim J-A, Scott R, Pires $\mathrm{CP}$, Paterson AH, Town C, Bancroft I: Comparative analysis between homoeologous genome segments of Brassica napus and its progenitor species reveals extensive sequence-level divergence. Plant Cell 2009, 21:1912-1928.

36. Lincoln S, Daly M, Lander E: Constructing genetic maps with MAPMAKER/EXP version 3.0: a tutorial and reference manual. Whitehead Inst Biomed Res Tech Rpt, 3rd edn. Cambridge: Whitehead Institute for Biomedical Research; 1993.

37. The Multinational Brassica Genome Project. http://www.brassica.info/resource/ maps/lg-assignments.php.

38. Windows QTL Cartographer 2.5. http://statgen.ncsu.edu/qtlcart/WQTLCart. htm.

39. Schuler G: Sequence mapping by electronic PCR. Genome research 1997 7:541-550.

40. The Brassica Database. http://brassicadb.org/brad/index.php.

41. The Brassica oleracea Genome Database. http://www.ocri-genomics.org/ bolbase/.

42. Altschul S, Madden T, Schäffer A, Zhang J, Zhang Z, Miller W, Lipman D: Gapped BLAST and PSI-BLAST: a new generation of protein database search programs. Nucleic acids research 1997, 25:3389-3402.

43. The Arabidopsis Information Resource. http://www.arabidopsis.org/.

44. Sambrook J, Russell DW: Molecular Cloning: A Laboratory Manual. 3rd edition. New York: Cold Spring Harbor Laboratory Press; 2001.

45. Parkin IAP, Sharpe AG, Keith DJ, Lydiate DJ: Identification of the A and C genomes of amphidiploid Brassica napus (oilseed rape). Genome 1995, 38:1122-1131.

46. Lowe AJ, Moule C, Trick M, Edwards KJ: Efficient large-scale development of microsatellites for marker and mapping applications in Brassica crop species. Theor Appl Genet 2004, 108:1103-1112.

47. Yang QY, Fan CC, Guo ZH, Qin J, Wu JZ, Li QY, Fu TD, Zhou YM: Identification of FAD2 and FAD3 genes in Brassica napus genome and development of allele-specific markers for high oleic and low linolenic acid contents. Theor Appl Genet 2012, 125:715-729.

48. Frary A, Nesbitt TC, Frary A, Grandillo S, Knaap E, Bin C, Liu J, Meller J, Elber R, Alpert KB, Tanksley SD: fw2.2: a quantitative trait locus key to the evolution of tomato fruit size. Science 2000, 289:85-88.

49. Steffen J, Kang I, Portereiko M, Lloyd A, Drews G: AGL61 interacts with AGL80 and is required for central cell development in Arabidopsis. Plant Physiol 2008, 148:259-268.

50. Cheng W, Taliercio E, Chourey P: The Miniaturel seed locus of maize encodes a cell wall invertase required for normal development of endosperm and maternal cells in the pedicel. Plant Cell 1996, 8:971-983.

51. Kang I, Steffen J, Portereiko M, Lloyd A, Drews G: The AGL62 MADS domain protein regulates cellularization during endosperm development in Arabidopsis. Plant Cell 2008, 20:635-647.
52. Becraft $P$, Stinard $P$, McCarty D: CRINKLY4: A TNFR-like receptor kinase involved in maize epidermal differentiation. Science 1996, 273:1406-1409.

53. Hutchison C, Li J, Argueso C, Gonzalez M, Lee E, Lewis MW, Maxwell BB, Perdue T, Schaller G, Alonso J: The Arabidopsis histidine phosphotransfer proteins are redundant positive regulators of cytokinin signaling. Plant Cell 2006, 18:3073-3087.

54. Song $X$, Huang W, Shi M, Zhu MZ, Lin HX: A QTL for rice grain width and weight encodes a previously unknown RING-type E3 ubiquitin ligase. Nat Genet 2007, 39:623-630.

55. Fan CC, Xing YZ, Mao HL, Lu T, Han B, Xu CG, Li XH, Zhang QF: GS3, a major QTL for grain length and weight and minor QTL for grain width and thickness in rice, encodes a putative transmembrane protein. Theor Appl Genet 2006, 112:1164-1171.

56. Weng J, Gu S, Wan X, Gao H, Guo T, Su N, Lei C, Zhang X, Cheng Z, Guo X, Wang J, Jiang $L$, Zhai $H$, Wan J: Isolation and initial characterization of GW5, a major QTL associated with rice grain width and weight. Cell Research 2008, 18:1199-1209.

57. Shomura A, Izawa T, Ebana K, Ebitani T, Kanegae H, Saeko KS, Masahiro YM: Deletion in a gene associated with grain size increased yields during rice domestication. Nat Genet 2008, 40:1023-1028.

58. Wang E, Wang J, Zhu X, Hao W, Wang L, Li Q, Zhang L, He W, Lu B, Lin H, $\mathrm{Ma} H$, Zhang $\mathrm{G}, \mathrm{He} \mathrm{Z}$ : Control of rice grain-filling and yield by a gene with a potential signature of domestication. Nat Genet 2008, 40:1370-1374

59. Luo M, Dennis ES, Berger F, Peacock WJ, Chaudhury A: MINISEED3 (MINI3), a WRKY family gene, and HAIKU2 (IKU2), a leucine-rich repeat (LRR) KINASE gene, are regulators of seed size in Arabidopsis. Proc Natl Acad Sci USA 2005, 102:17531-17536.

60. Mizukami Y, Fischer R: Plant organ size control: AINTEGUMENTA regulates growth and cell numbers during organogenesis. Proc Natl Acad Sci USA 2000, 97:942-947.

61. Jofuku KD, Omidyar PK, Gee Z, Okamuro JK: Control of seed mass and seed yield by the floral homeotic gene APETALA2. Proc Natl Acad Sci USA 2005, 102:3123-3128.

62. Deng Y, Dong H, Mu J, Ren B, Zheng B, Ji Z, Yang W, Liang Y, Zuo J: Arabidopsis histidine kinase CKI1 acts upstream of HISTIDINE PHOSPHOTRANSFER PROTEINS to regulate female gametophyte development and vegetative growth. Plant Cell 2010, 22:1232-1248.

63. Wang A, Garcia D, Zhang H, Feng K, Chaudhury A, Berger F, Peacock WJ, Dennis ES, Luo M: The VQ motif protein IKU1 regulates endosperm growth and seed size in Arabidopsis. Plant J 2010, 63:670-679.

64. Saze $H$, Kakutani T: Heritable epigenetic mutation of a transposon-flanked Arabidopsis gene due to lack of the chromatin-remodeling factor DDM1. The EMBO Journal 2007, 26:3641-3652

65. Canales C, Bhatt A, Scott R, Dickinson H: EXS, a putative LRR receptor kinase, regulates male germline cell number and tapetal identity and promotes seed development in Arabidopsis. Curr Biol 2002, 12:1718-1727.

66. Zhou Y, Zhang X, Kang X, Zhao X, Zhang X, Ni M: SHORT HYPOCOTYL UNDER BLUE1 associates with MINISEED3 and HAIKU2 promoters in vivo to regulate Arabidopsis seed development. Plant Cell 2009, 21:106-117.

67. Pien S, Grossniklaus U: Polycomb group and trithorax group proteins in Arabidopsis. Biochim Biophys Acta 2007, 1769:375-382.

68. Schruff M, Spielman M, Tiwari S, Adams S, Fenby N, Scott R: The AUXIN RESPONSE FACTOR 2 gene of Arabidopsis links auxin signaling, cell division, and the size of seeds and other organs. Development 2005, 133:251-261.

69. Luo M, Bilodeau P, Dennis E, Peacock W, Chaudhury A: Expression and parent-of-origin effects for FIS2, MEA, and FIE in the endosperm and embryo of developing Arabidopsis seeds. Proc Natl Acad Sci USA 2000, 97:10637-10642

70. Garcia D, Fitz Gerald JN, Berger F: Maternal control of integument cell elongation and zygotic control of endosperm growth are coordinated to determine seed size in Arabidopsis. Plant Cell 2005, 17:52-60.

71. Roxrud I, Lid S, Fletcher J, Schmidt E, Opsahl-Sorteberg H: GASA4, one of the 14-member Arabidopsis GASA family of small polypeptides, regulates flowering and seed development. Plant Cell Physiol 2007, 48:471-483.

72. Riefler M, Novak O, Strnad M, Schmülling T: Arabidopsis cytokinin receptor mutants reveal functions in shoot growth, leaf senescence, seed size, germination, root development, and cytokinin metabolism. Plant Cell 2006, 18:40-54. 
73. FitzGerald J, Luo M, Chaudhury A, Berger F: DNA methylation causes predominant maternal controls of plant embryo growth. PLoS One 2008, 3(5):e2298.

74. Kondou Y, Nakazawa M, Kawashima M, Ichikawa T, Yoshizumi T, Suzuki K, Ishikawa A, Koshi T, Matsui R, Muto S, Matsui M: Retarded Growth Of Embryo1, a new basic helix-loop-helix protein, expresses in endosperm to control embryo growth. Plant Physiol 2008, 147:1924-1935.

75. Takahashi N, Nakazawa M, Shibata K, Yokota T, Ishikawa A, Suzuki K, Kawashima M, Ichikawa T, Shimada H, Matsui M: shk1-D, a dwarf Arabidopsis mutant caused by activation of the CYP72C1 gene, has altered brassinosteroid levels. Plant J 2005, 42:13-22.

76. Li Y, Zheng L, Corke F, Smith C, Bevan MW: Control of final seed and organ size by the DA1 gene family in Arabidopsis thaliana. Genes \& Dev 2008, 22:1331-1336.

77. Portereiko M, Lloyd A, Steffen J, Punwani J, Otsuga D, Drews G: AGL80 is required for central cell and endosperm development in Arabidopsis. Plant Cell 2006, 18:1862-1872.

doi:10.1186/1471-2156-13-105

Cite this article as: Cai et al: Identification of candidate genes of QTLs for seed weight in Brassica napus through comparative mapping among Arabidopsis and Brassica species. BMC Genetics 2012 13:105.

\section{Submit your next manuscript to BioMed Central and take full advantage of:}

- Convenient online submission

- Thorough peer review

- No space constraints or color figure charges

- Immediate publication on acceptance

- Inclusion in PubMed, CAS, Scopus and Google Scholar

- Research which is freely available for redistribution 